\title{
Information Processing by Pigeons (Columba livia): Incentive as Information
}

\author{
Charles P. Shimp and Alyson L. Froehlich \\ University of Utah
}

\author{
Walter T. Herbranson \\ Whitman College
}

\begin{abstract}
Experiment 1 showed that the Hick-Hyman law (W. E. Hick, 1952; R. Hyman, 1953) described the effects of anticipated reinforcement, a form of incentive, on pigeons' (Columba livia) reaction time to respond to a target spatial location. Reaction time was an approximately linear function of amount of information interpreted as probability of reinforcement, implying that pigeons processed incentive at a constant rate. Experiment 2 showed that the Hick-Hyman law described effects of incentive even when it varied from moment to moment in a serial reaction time task similar to that of M. J. Nissen and P. Bullemer (1987), and processing information about target spatial location modulated absolute reaction time and not rate of processing incentive. The results support mental continuity and provide comparative support for the idea of the economics of information in economic theory about the incentive value of information.
\end{abstract}

Keywords: incentive, anticipated reinforcement, Hick-Hyman law, reaction times, pigeons
"Information . . . is a source of pleasure . . . in its own right. This has diverse consequences for human decision making" (Loewenstein, 2006, p. 705). The present experiments suggest that information about the probability of anticipated reinforcement has a hedonic value that can, by itself, also guide some nonhuman animal performances, in the present case the key-pecking behavior of pigeons. To see that this is so, let us begin by reviewing some basic facts about information. Chase (1978) stated that "the number of choices, their probability, and their temporal and spatial predictability all cause uncertainty. ... One of the basic tenets of human performance is that man is slow when he is uncertain" (p. 25). In some special cases, the time it takes a human participant to respond to stimuli is, in fact, a linear function of the amount of information those stimuli provide (Hick, 1952; Hyman, 1953). Reaction time in these cases is interpreted to reflect the number of implicit binary decisions a participant processes sequentially, at a constant rate, before responding to a target location. This information processing interpretation of the linear function is known as the Hick-Hyman law (Wickens \& Hollands, 2000).

Vickrey and Neuringer (2000) and Wasserman and his colleagues (e.g., Young \& Wasserman, 1997) have suggested that the theory of human information processing applies more extensively

Charles P. Shimp and Alyson L. Froehlich, Department of Psychology, University of Utah; Walter T. Herbranson, Department of Psychology, Whitman College.

We thank Sibel Iren, Taylor Neville Johnson, Jason Nigbuhr, Elizabeth Thatcher, and Krystyl White, whose help in running birds was essential to this research. We also thank Dave Strayer and Thane Fremouw for enlightening conversations about and literature references to human information processing.

Correspondence concerning this article should be addressed to Charles P. Shimp, Department of Psychology, 380 South, 1530 East Room 502, University of Utah, Salt Lake City, UT 84112-0251. E-mail: charlie.shimp@psych.utah.edu to animal cognition than has been widely appreciated. Vickrey and Neuringer (2000) varied the number of possible target locations to which a pigeon was required to respond in a task closely resembling the original human tasks in which the Hick-Hyman law was discovered and found that the law described their results: Reaction time was a linear function of the amount of information processed about spatial target location.

The amount of information conveyed by a stimulus can be expressed in terms of either a probability or a relative-frequency estimate of a probability. The formal relation between information in bits, $H_{s}$, and probability is

$$
H_{s}=\log _{2}(1 / P),
$$

where $P$ is the probability of occurrence of some event (Welford, 1968; Wickens \& Hollands, 2000, p. 45). In this article, information refers, via Equation 1, to the probability or relative frequency of an event consisting of the delivery of a reinforcer. Equation 1, combined with the Hick-Hyman law, provides a basis for using reaction time as a function of relative frequency to determine the rate of processing statistical information in terms of the number of bits processed per second (Hick, 1952; Hyman, 1953; Welford, 1968; Wickens \& Hollands, 2000). More generally, the rate of processing statistical information from this perspective is simply the slope of the function relating reaction time to probability. Note that absolute reaction time by itself does not reflect rate of processing because, as Chase (1978) noted, reaction time generally reflects uncertainty, but reaction time can be short, moderate, or long given the same amount of uncertainty, depending on the nature of the response, the task, the subject, and other variables (Sternberg, 1969). Salient among these variables are payoff, motivation, and incentive (Garner, 1974; Vickrey \& Neuringer, 2000). Incentive may affect reaction time but have little or no effect on the rate at which humans process information about such variables as target spatial location. Rate of processing accordingly has sometimes been viewed as reflecting a person's fixed band- 
width or channel capacity, which is relatively resistant to manipulation by motivational variables (Eysenck, 1987; Jensen, 1987). Fitts and his colleagues (e.g., Bahrick, Fitts, \& Rankin, 1952) varied payoffs, manipulated speed-accuracy trade-offs, and used information theory to show that although response speed depended on incentive, rate of processing other variables varied little (Bahrick et al., 1952; Brainard, Irby, Fitts, \& Alluisi, 1962).

\section{Experiment 1}

We sought to generalize the capability of information theory to describe the effects of incentive. Previous human research and the corresponding pigeon research by Vickrey and Neuringer (2000) has shown that incentive might have little or no effect on the rate at which information about other variables, such as target spatial location, is processed, but this previous research says nothing about how incentive itself is processed. We therefore ask whether incentive itself is processed in accordance with the Hick-Hyman law. Thus, in Experiment 1 we sought to determine whether information theory could reveal that pigeons process incentive in a manner such that response speed is a linear function of the amount of information about incentive required to be processed.

By incentive, we mean anticipated reinforcement. We do not claim either that all reinforcement involves anticipation or that all forms of incentive involve reinforcement. The terminology is justified because we examine the possibility that reaction time reflects an organism's anticipation of a future event (Froehlich, Herbranson, Loper, Wood, \& Shimp, 2004), that this anticipation involves processing of statistical information, and, given that this information is about reinforcement, that the processing of that information might be motivating and, in plain English, tend to incite an organism to action (Simpson \& Weiner, 1989).

\section{Method}

Participants. The pigeons (Columba livia) had served previously in Experiment 3 in Froehlich et al. (2004). Table 1 shows that 5 birds began the replication. Bird 1 was excluded from the replication beginning in Condition 7 because it stopped responding when the reinforcement probability became extremely low, and Bird 5 had to be euthanized because of illness in Condition 8. Each bird was maintained at approximately $80 \%$ of its free-feeding weight, with supplemental Western Sun Pigeon Feed provided as

Table 1

Experimental Conditions in Experiment 1

\begin{tabular}{cccc}
\hline Condition no. & No. days & No. birds & Reinforcement probability \\
\hline 1 & 20 & 5 & .0100 \\
2 & 20 & 5 & .1000 \\
3 & 20 & 5 & .0250 \\
4 & 22 & 5 & .1000 \\
5 & 20 & 5 & .0500 \\
6 & $14^{\mathrm{a}}$ & 5 & .0250 \\
7 & 20 & 4 & .0150 \\
8 & 20 & 3 & .0100 \\
9 & 20 & 3 & .0075 \\
\hline
\end{tabular}

${ }^{a}$ A change from one experimenter to another caused this condition to be run only 14 days. needed following daily experimental sessions. The birds were housed individually in standard pigeon cages with free access to water and grit in a colony room with a 14:10 light-dark cycle. All sessions took place at approximately the same time 4 to 6 days a week.

Apparatus. The apparatus was identical to that in Froehlich et al. (2004). Five standard three-key LeHigh Valley Electronics (Laurel, MD) pigeon chambers were multiplexed to an IBM PS/2 Model 95 computer, which arranged all the experimental contingencies and recorded the data. The three keys were arranged in a horizontal row approximately $24 \mathrm{~cm}$ above the floor. The center key was located directly above the food hopper. The centers of the left and right keys were approximately $8 \mathrm{~cm}$ to the left and right of the center of the middle key. Reinforcement consisted of access to mixed grain presented in the food hopper. Ventilator fans and white noise helped to mask extraneous sounds. A houselight remained on during the entire experiment, except during reinforcement.

Procedure. There were 750 discrete trials per daily session. Each trial began with the illumination of a single key, which remained illuminated until pecked. A single peck either initiated a 0.5 -s response-to-stimulus interval or delivered a reinforcer consisting of access to mixed grain. Reinforcer duration was $2 \mathrm{~s}$. Pecks at dark keys had no programmed consequences and were not recorded. Experimental conditions were run for 20 days.

In each condition, only one key was lit per trial, but the location of the lit key on each trial was randomly selected, with equal probabilities, from left, center, or right. Each peck to an illuminated key was reinforced according to a probability that varied over experimental conditions but that remained constant within a condition. We sought to determine whether the effects of anticipated reinforcement probability, or incentive, could be described by the Hick-Hyman law. In particular, we asked whether increasing anticipated reinforcement probability could be interpreted as decreasing the amount of information to be processed. If so, then reaction time should be an approximately linearly decreasing function of reinforcement probability. Table 1 shows how reinforcement probability was varied across conditions.

\section{Results}

The left panel of Figure 1 shows mean median reaction time, averaged over the 3 to 5 birds (see Table 1) and the last 5 days of a condition, plotted as a function of the probability of reinforcement. The right panel shows the same data plotted as a function of amount of information. Figure 1 suggests that response speed was an approximately linear function of amount of information. An analysis of variance (ANOVA; Edwards, 1979) confirmed a significant linear component, $F(1,21)=6.166, p<.05$ (effect size, $r=.618$; Rosnow \& Rosenthal, 2003), and an ANOVA confirmed that there was no significant nonlinear deviation, $F(4,21)=1.336$, $p<.50$ (effect size, $r=.344$ ). The function in Figure 1 is shallow, but an ANOVA confirmed that its slope was reliably different from zero, $F(1,38)=7.783, p<.01$ (effect size, $r=.412$ ). Thus, the results clearly show that greater incentive linearly increased response speed in accordance with the Hick-Hyman law. 

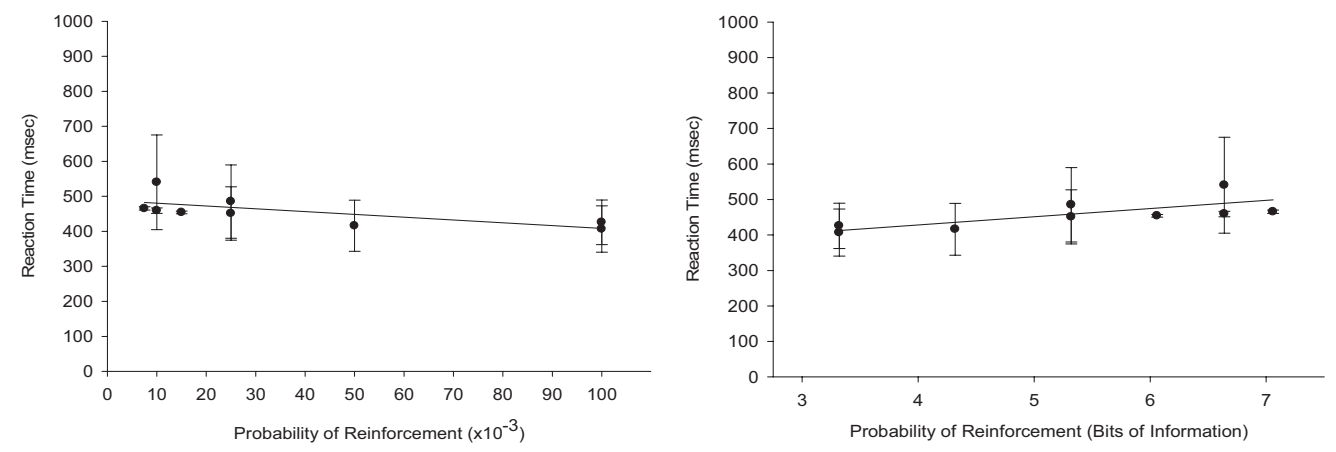

Figure 1. Mean median reaction time, averaged over the 3 to 5 birds (see Table 1) and the last 5 days of a condition in Experiment 1, plotted in the left panel as a function of the probability of reinforcement when a pigeon was required to peck a lit key that randomly varied from trial to trial over three keys. Vertical bars show 95\% confidence intervals. The linear regression line had a slope and intercept of -801 and 488, respectively. The value of $R^{2}$ was .17. The right panel shows the same data as plotted in the left panel, but plotted as a function of the uncertainty of reinforcement (number of bits). Estimated slope and intercept were 23 and 336, respectively. Information theory predicts a negative slope in the left panel and a correspondingly positive slope in the right panel.

\section{Discussion}

The Hick-Hyman law has been used previously to show that incentive has relatively little effect on the processing of other kinds of information. Experiment 1 uses the Hick-Hyman law to show that incentive itself is information that is processed just like other kinds of information. Pigeons processed information in the form of incentive, or anticipated reinforcement, such that reaction time was a linear function of the amount of information required to be processed.

\section{Experiment 2}

The effect of incentive in Experiment 1 was reliable, but the magnitude of the effect was numerically relatively small: Response speed changed relatively little as the amount of information was varied. Information theory interprets this kind of relatively flat function as reflecting a relatively fast rate of processing information: A flatter function reflects that a subject requires relatively less time to process the same increment in information. Why was the rate of information processing in Experiment 1 so fast? The task in Experiment 1 did not change the information processing requirements over time, either from trial to trial within a session or across days within a condition. We conjecture that rate of processing was very fast because once a pigeon learned an estimate of the probability of a target location or of reinforcement, the task did not demand any reestimations; the pigeon needed only to continue to keep an estimate active in working memory.

On this hypothesis, Experiment 1 described an "easy" case of information processing and did not provide an especially accurate estimate of the pigeon's channel capacity. In most naturalistic tasks, probabilities need to be updated and reestimated from moment to moment. For this reason, some of the earliest applications of information theory in psychology were actually to sequential performances in which information processing demands varied from moment to moment in the context of serial patterns of stimuli (Bennett, Fitts, \& Noble, 1954). The hope was that information theory would offer a way to characterize the dynamics of the behavior stream, ultimately including the dynamics of speech (Frick \& Miller, 1949; Miller \& Frick, 1951). Related ideas persist today; it has been assumed that the capacity to segment continuous speech sounds into distinct component words depends in part on the rapid processing of statistical information by highly dynamic, nonlinguistic, statistical learning mechanisms (Saffran, 2003) There is no doubt, in other words, that humans can and routinely do track statistical information that varies from moment to moment.

A variety of evidence hints that pigeons can also estimate momentary statistical information, including the momentary likelihood of reinforcement (e.g., Hinson \& Staddon, 1983; Shimp, 1966). It would not surprise us if this capability were extremely general, because, in a sense, virtually everything an organism does has immediate consequences that depend on how well it exploits information about the immediate past. If a person says things that do not mesh with his or her immediately preceding speech, no one will respond approvingly; if a pianist plays notes that do not sensibly follow previous notes, an audience will disapprove; and if a child learning to walk places weight on his or her left foot and ignores the resulting appropriate rebalancing, he or she is more likely to fall down and not meet with the social approval of his or her parents. In this sense, people routinely engage in and expect from others a kind of continuity in performance, whereby information about the immediate past serves as a guide to what consequences to expect in the immediate future.

Therefore, in Experiment 2 we sought to determine whether pigeons also have this capacity to process rapidly changing statistical information about anticipated consequences, in particular about anticipated reinforcement. Experiment 2 asked whether the Hick-Hyman law would describe the effects of highly dynamic information about incentive as it did corresponding static information in Experiment 1. We predicted that if it did, the slope of the function relating reaction time to information would be steeper, would reflect the greater information processing demands required in this experiment than in Experiment 1, and hence would more accurately reflect the pigeon's channel capacity. 
To determine how rapidly changing incentive is processed, we adapted a serial reaction time task developed by Nissen and Bullemer (1987). They instructed a participant to press a key that corresponded to an illuminated spatial location. Over trials, the illuminated location varied across several possible locations. Reaction time was measured from the onset of the light at a spatial location to a participant's response to the corresponding key. For one group, the lights appeared in a repeating 10-item list, and for another group, the lights appeared in a random order. Participants in the group with a structured list responded, on the average, faster than the participants in the random group, which implies that participants learned something about the structure of the repeating list. Curran, Smith, DiFranco, and Daggy (2001) and Hunt and Aslin (2001) further demonstrated that the local structure of a list affects local reaction times: The greater the likelihood of a target spatial location is, given the previous one or two locations, the faster is responding to that location.

Herbranson (2000) invented an avian version of Nissen and Bullemer's (1987) serial reaction time task. Froehlich et al. (2004) further developed Herbranson's task and found that their pigeons' reaction times were similar in some detail to those in the human experiments by Curran et al. (2001) and Hunt and Aslin (2001): Reaction time in both pigeon and human serial reaction time experiments was an approximately linear function of the local likelihood of a target spatial location. Using Equation 1, we can conclude that Froehlich et al. (2004) showed that the Hick-Hyman law describes how pigeons process dynamically changing information about target spatial likelihood.

Experiment 2 therefore adapted the serial reaction time task of Froehlich et al. (2004) to determine how pigeons process rapidly changing incentive. Specifically, we asked whether response speed is a linear function of momentary incentive - that is, we asked whether the Hick-Hyman law describes how pigeons process local incentive.

\section{Method}

Participants. Four male White Carneaux pigeons (Columba livia) were obtained from the Palmetto Pigeon Plant (Sumter, SC). Two were experimentally naïve, and 2 had previously served in experiments on multidimensional categorization.

Apparatus. The apparatus was as described for Experiment 1.

Procedure. The basic procedure was similar to that of Froehlich et al. (2004). There were 750 discrete trials per daily session. Each trial consisted of the illumination of one of the three white keys and a corresponding peck to that key. The single illuminated key remained illuminated until pecked once. A single peck initiated either a 0.5 -s response-to-stimulus interval or a reinforcer consisting of 2-s access to mixed grain. The three keys were, on the average, equally likely to be illuminated. Each peck to an illuminated key was reinforced according to a random ratio schedule that was varied across serial positions in the list described below. Pecks at dark keys had no programmed consequences and were not recorded.

Each of the two conditions involved the same repeating nineitem sequence of singly lit left, center, or right keys. The list was as follows: left, center, right, left, right, center, right, left, center. (This list is the same as the list used in Condition 11 of Experiment 1 in Froehlich et al., 2004.) After the ninth serial position, the entire nine-item list was repeated, and it continued to repeat until 750 items had been presented. Daily sessions began at randomly selected, equally likely serial positions.

This sequence provided two different values of first-order local predictability, .33 and .67 . The first-order local predictability of a spatial location was the relative frequency of the illumination of the key in that location, conditionalized on the previous location. For example, in the list used here, a lit left key given a previously lit center key occurred once out of the three occasions on which center occurred. The relative frequency of left given a previous center was therefore .33. Correspondingly, left followed right two out of the three occasions on which right occurred, so that the relative frequency of left given right was .67. All other possibilities were computed in a similar manner. The sequence also provided two different values of second-order local predictability, .50 and 1.00. The second-order local predictability of a spatial location was the relative frequency of the illumination of the key in that location, conditionalized on the previous two locations. For example, a lit left key followed a previous sequence of center followed by right on both occasions when center was followed by right. The relative frequency of left given a previous center-right pair was therefore 1.00. All other possible second-order local values of predictability of a target location were computed in a similar manner.

Table 2 shows how the local probability of reinforcement was varied as a function of serial position. In Condition 2, the local random ratio schedules for serial positions that had random ratio 10 and extinction were the reverse of those in Condition 1. All birds ran first in Condition 1 and then in Condition 2. The overall reinforcement probability per key peck was maintained at approximately the same value, .05, as in Froehlich et al. (2004). Experimental conditions were run for 20 days.

\section{Results}

Reaction times to the first nine locations in a session were omitted from the analysis to reduce the impact of any warm-up

Table 2

Experimental Conditions in Experiment 2

\begin{tabular}{cccccccccc}
\hline & \multicolumn{8}{c}{ Local Reinforcement Probability for Nine Serial Positions in the List } \\
\cline { 2 - 10 } Condition & Left & Center & Right & Left & Right & Center & Right & Left & Center \\
no. & 1 & 2 & 3 & 4 & 5 & 6 & 7 & 8 & 9 \\
\hline 1 & .05 & .00 & .05 & .10 & .10 & .00 & .05 & .10 & .00 \\
2 & .05 & .10 & .05 & .00 & .00 & .10 & .05 & .00 & .10 \\
\hline
\end{tabular}


effect, and, similarly, reaction times that occurred to the first nine items after reinforcement were omitted to reduce the impact of postreinforcement pauses. We examined asymptotic performance in terms of the two separable components of information processing by pigeons in the serial reaction time task, response speed and rate of information processing.

The left panel of Figure 2 shows mean median reaction time averaged over the 4 birds, the last 5 days of a condition, and all serial positions in either of the two experimental conditions that had a specified local probability of reinforcement, as shown in Table 2. For example, for a local probability of reinforcement of .10 , Figure 2 shows the mean median reaction times averaged over Serial Positions 4, 5, and 8 in Condition 1 and Serial Positions 2, 6, and 9 in Condition 2. Figure 2 suggests that response speed was an approximately linearly decreasing function of local reinforcement probability; local reaction times became shorter as the local probability of reinforcement increased. Figure 2 also shows the linear regression line for the 12 individual-bird averages, one data point for each of 4 birds for each of three reinforcement probabilities (see legend for Figure 2 for estimated parameters). An ANOVA confirmed that there was a significant linear trend, $F(1,9)=6.609, p<.05$ (effect size, $r=.673$ ), and no significant deviation from linearity, $F(1,9)=0.007, p<.936$ (effect size, $r=.030$ ). (In this case, with three values of the independent variable, nonlinearity simply refers to a quadratic trend.) An ANOVA to test the hypothesis that the slope of the regression line was zero rejected the hypothesis and confirmed the results of visual inspection, $F(1,10)=7.241, p<.025$ (effect size, $r=.648$ ). In summary, Figure 2 shows that response speed was a linearly decreasing function of local anticipated reinforcement (i.e., incentive), so that the Hick-Hyman law describes how momentary incentive was processed.

The right panel of Figure 2 shows the same data plotted after Equation 1 was used to convert probability to amount of information, or number of bits. The value of zero for the local probability of reinforcement produced a singularity, so the regression line is not plotted for all three points in the right panel. The right panel shows that response speed was an increasing function of the amount of uncertainty: Speed of responding became slower as local uncertainty increased.

Figure 3 shows how the results in Figure 2 are averages over different local likelihoods of target spatial locations. The left panels of Figure 3 show mean median reaction time as a function of local incentive, defined as local probability of reinforcement, averaged over the serial positions with the specified reinforcement probability. The averages are also over the 4 birds and the last 5 days of a condition for each of the two separate values of local predictability of target locations. The top left panel shows reaction time for each of the two possible values, .33 and .67 , of first-order local predictability of a target location, and the bottom left panel shows reaction time for each of the two possible values, .50 and 1.00 , of second-order local predictability. The right panels of Figure 3 show the same data with reaction time plotted as a function of amount of information. Visual inspection of Figure 3 suggests that response speed was a linear function of the local probability of reinforcement and that the slope of the function was perhaps statistically similar for different local likelihoods of target locations. The slopes for first-order functions appear slightly different in the upper left panel, but the variability suggests that this is not reliable. In short, visual inspection of Figure 3 suggests that (a) response speed increased linearly as local incentive increased and (b) rate of processing local incentive might have been approximately constant for two different values of predictability of target locations.

Statistical analyses confirmed both these phenomena. First, response speed reliably depended on incentive when first-order local likelihood was higher, at .67, $F(1,10)=8.519, p<.05$ (effect size, $r=.678)$, although not when first-order local predictability of a target location was lower, at .33, $F(1,10)=4.493, p>.05$ (where $p_{.05}=4.964$; effect size, $r=.557$ ). Thus, greater local incentive reliably increased response speed at one of the two values of first-order local target location predictability, and even when it did not, there was an appreciable effect size. Response speed depended on incentive for both values, .50 and 1.00 , of
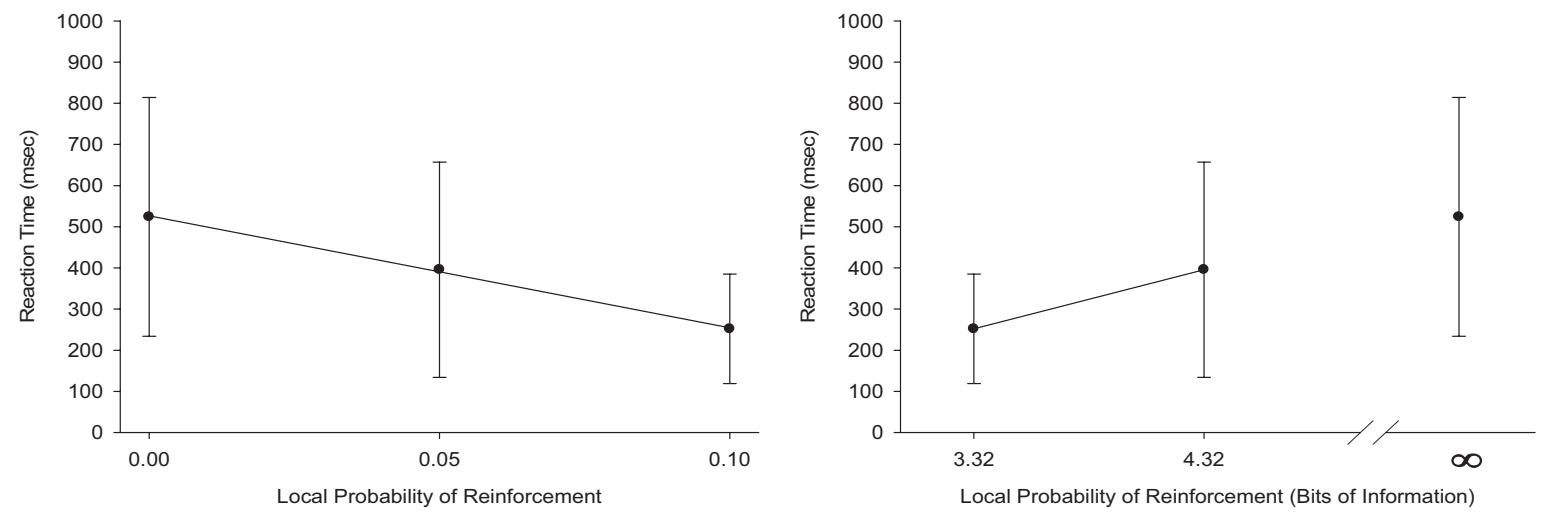

Figure 2. Local mean median reaction time averaged over 4 birds and the last 5 days of a condition, plotted in the left panel as a function of the local probability of reinforcement, in Experiment 2. Vertical bars show 95\% confidence intervals. Also shown is the linear regression line, with slope of $-2,720$, intercept of 526 , and $R^{2}$ of .42. The same data are plotted in the right panel in terms of amount of information (number of bits). Information theory predicts a negative slope in the left panel and a correspondingly positive slope in the right panel. 

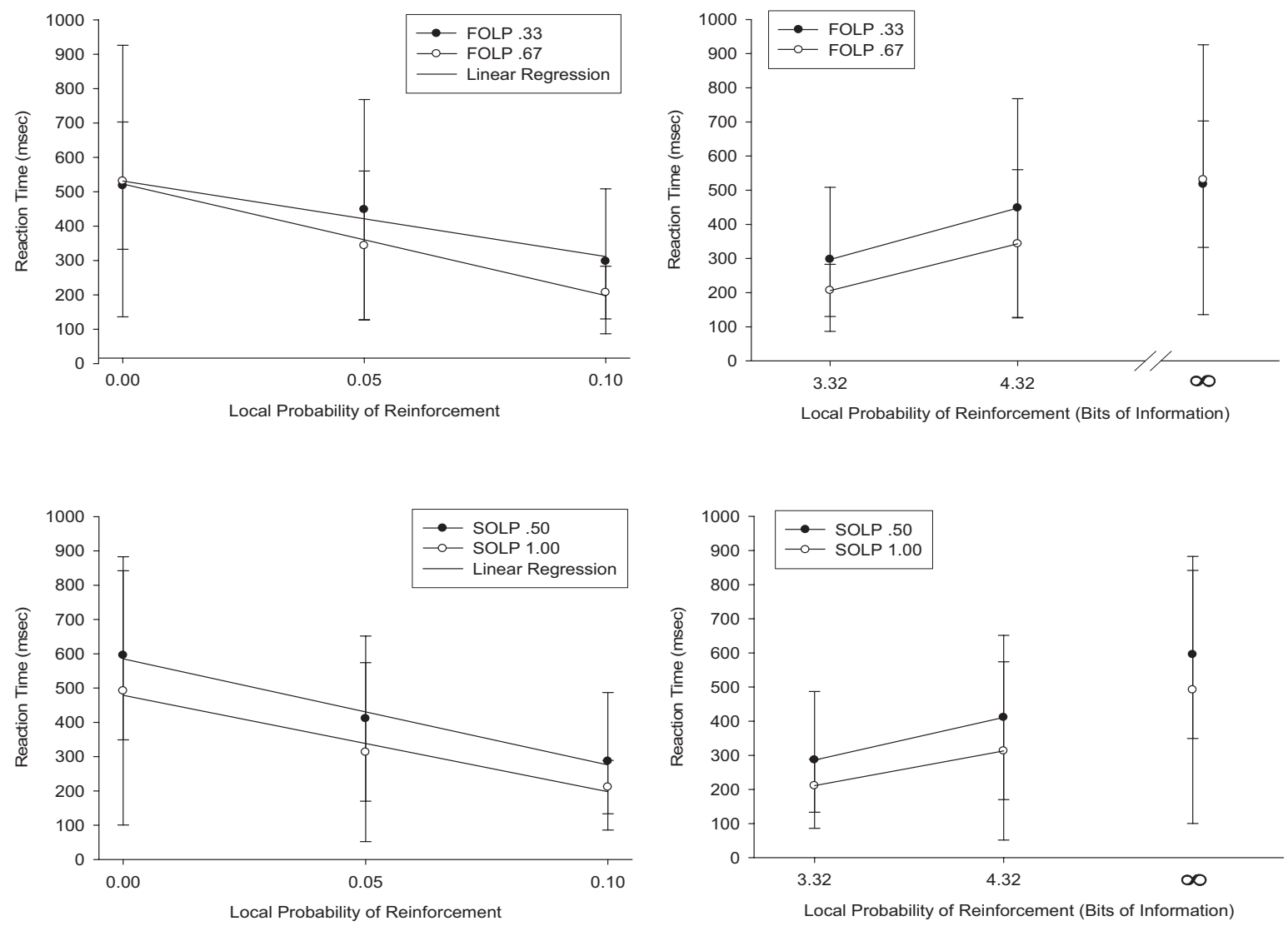

Figure 3. Local mean median reaction time averaged over the 4 birds and the last 5 days of a condition, plotted as a function of the local probability of reinforcement, for each of the two values (.33 and .67) of first-order local predictability (FOLP) of a target location in Experiment 2. Vertical bars show 95\% confidence intervals. Also shown are the corresponding linear regression lines with slopes of $-2,199$ and $-3,242$, intercepts of 531 and 522 , and $R^{2}$ of .31 and .46 , respectively (top left panel). The same data are plotted in terms of amount of information (number of bits) in the top right panel. The corresponding functions for each of the two values (.50 and 1.00) of second-order local predictability (SOLP) of a target location with linear regression slopes of $-3,091$ and $-2,806$, intercepts of 585 and 479 , and $R^{2}$ of .50 and .37 , respectively, are shown in the bottom left panel. The same data are plotted in terms of amount of information in the bottom right panel.

second-order local predictability of a target location: $F(1,10)=$ $10.000, p<.05$ (effect size, $r=.707$ ), for $.50 ; F(1,10)=5.873$, $p<.05$ (effect size, $r=.608$ ), for 1.00. In summary, response speed reliably depended on three of the four values of first- and second-order local predictability of target spatial location.

Reaction time was generally a linear function of local incentive. There was a linear trend approaching significance for first-order local predictability equal to $.33, F(1,9)=4.049, p<$ .075 (effect size, $r=.557$ ), and no reliable deviation from linearity, $F(1,9)=0.184, p<.678$ (effect size, $r=.142$ ). Correspondingly, there was a significant linear trend for firstorder local predictability equal to $.67, F(1,9)=7.639, p<.025$ (effect size, $r=.678$ ), and no reliable deviation from linearity, $F(1,9)=0.062, p<.809$ (effect size, $r=.083$ ). A pattern even more compatible with linearity emerged for the case of secondorder local predictability values of .50 and 1.00 , for which both linear trends were significant and neither deviation from linearity was significant. There was a significant linear trend for second-order local predictability equal to $.50, F(1,9)=9.122$, $p<.025$ (effect size, $r=.709$ ), and no reliable deviation from linearity, $F(1,3)=0.116, p<.741$ (effect size, $r=.113$ ). Correspondingly, there was a significant linear trend for second-order local predictability equal to $1.00, F(1,9)=5.261$, $p<.05$ (effect size, $r=.607$ ), and no reliable deviation from linearity, $F(1,9)=0.132, p<.725$ (effect size, $r=.120$ ).

Second, there was no reliable evidence that the rate of processing incentive depended on either first- or second-order local predictability of the target spatial location. The slopes of the two regression lines for first-order local likelihood of a target location were not reliably different, $t(2)=0.264, p>.50$, and the effect size ( $r=.184)$ was relatively small; similarly, the two corresponding slopes for second-order local likelihood of a target location also gave no evidence that the slopes were different, $t(2)=0.068, p>.50$, and the effect size $(r=.048)$ was very small. Thus, there is no evidence that the rate of processing local incentive depended on either first- or secondorder target location likelihood. The rate of processing momentary incentive was approximately a constant. 


\section{Discussion}

The Hick-Hyman law described how pigeons tracked momentary incentive in the form of rapidly changing statistical information about anticipated reinforcement. Pigeons therefore appeared to process momentary information about reinforcement by making a sequence of implicit binary choices at a constant rate. The results confirm our speculation that the rate at which these implicit binary choices were made in Experiment 2 would be slower than in Experiment 1. We had predicted that if the Hick-Hyman law were applicable, the slope of the regression line relating reaction time to information would be steeper than in Experiment 1, and it was. Although there is no reason to assume the specific conditions of Experiment 2 maximally taxed the pigeon's processing capacity, so that Experiment 2 directly revealed the pigeon's channel capacity, we believe it is safe to conclude that Experiment 2 demonstrates that increasing the rate at which a pigeon was required to track information increased the information-processing demands and provided a closer approximation to channel capacity than did Experiment 1. More generally, Experiment 2 demonstrates that information theory provides an excellent interpretative framework within which the effects of varying demands on processing capacity in nonhuman animals can be estimated.

\section{General Discussion}

\section{Incentive as Learned Information About Anticipated Reward}

Recall the quotation from Loewenstein (2006) with which we began, to the effect that information is a source of pleasure in its own right. This view provides an interesting contrast with a common interpretation in psychology of the relation between learning and motivation. Motivation is commonly viewed as qualitatively separate from associative learning. Accordingly, incentive is viewed as modulating either the strength of an associative connection or the likelihood that a given strength will suffice to generate performance (e.g., Dickinson \& Balleine, 2002; Hull, 1943; Tolman, 1932; Yerkes \& Dodson, 1908). An analogous two-process account asks how incentive modulates information processing (Eysenck, 1987; Jensen, 1987).

This two-process view integrates a huge literature and has much to commend it. However, it may not be a comprehensive account, because in Experiments 1 and 2, incentive itself had motivating properties: Incentive was information, was represented as amount of uncertainty in bits, and was processed according to the linear Hick-Hyman law. As far as we know, there is no reason for the classic two-process account to predict this linear function or to explain why it can be interpreted in terms of rate of processing bits of information. More generally, we think the present data defy several entire categories of learning and conditioning, such as statistical learning theory (Estes, 1958), the matching law (Herrnstein, 1997), and the Rescorla-Wagner model (Rescorla \& Wagner, 1972). There are several reasons why these theories fail to address the present results. First, the temporal properties of most learning and conditioning tasks on which these theories are based are not those that are required to reveal processing of local information (Froehlich et al., 2004). Second, theories predicting either the relative frequency of a response or response strength would have to add suitable auxiliary assumptions linking these variables to reaction time. Third, any theory involving strength of associations would have to invoke auxiliary assumptions to explain why the relation between reinforcement probability and reaction time is linear. Fourth, auxiliary assumptions about the dynamics of memory would be required to account for how first- and second-order local probability affected reaction time. Fifth, a reward-based theory would have to explain why rate of information processing was a constant, whereas response speed varied. This last problem historically was sufficiently difficult to solve that it was one of the main reasons why human cognitive psychologists switched allegiance from learning theory to information processing. In contrast with this awkward arrangement, information theory needs no new assumptions at all to describe our data.

Our results appear to be the first to reveal a context in which an animal's incentive is processed according to an elegantly simple form of information theory. From this perspective, a pigeon's processing of information in the form of incentive is no different from its processing of other kinds of information interpreted as uncertainty, including the spatial organization of visual stimuli (Young \& Wasserman, 1997) or the likelihood of a target's spatial location (Froehlich et al., 2004; Vickrey \& Neuringer, 2000).

Psychology is but one of several disciplines in which it is vital to understand how to conceptualize incentive. In economics, individuals are assumed to be motivated to obtain information to get a competitive advantage over others facing greater uncertainty. The illegality of insider trading of stocks tells us just how important the reduction of uncertainty can be and how strong the incentive function of information can be. Similarly, the incentive function of uncertainty is assumed to influence how governmental monetary and taxing policies affect income and economic growth (Bolton \& Dewatripont, 2005). More generally, the economics of information assumes that "people might lack information and be motivated to acquire it" (Loewenstein, 2006, p. 704; see also Laffont \& Martimor, 2001). We believe our results are the first to provide comparative support for this assumption.

How this incentive function of information has evolved is a key question in evolutionary biology and behavioral ecology. Maynard Smith and Harper (2003) theorized that foraging and choice behavior can be interpreted in terms of the motivating properties of information about anticipated events and thereby linked evolutionary theory, economic theory, and information theory. We believe our comparative results showing how the Hick-Hyman law describes processing of information in the form of incentive in a nonhuman animal species provide empirical support for a link among economic theory, evolutionary biology, and elementary information processing. We believe this link is probably stronger than some of the properties of our task directly suggest. Our tasks involved only information about anticipated positive outcomes. We suspect that an information processing analysis would also inform our understanding of the effects of anticipated negative outcomes. Perhaps, for example, the negative incentive of anticipated foods varying in toxicity, the negative incentive of anticipated appearances of a predator, or the positive incentive of anticipated palatable foods would submit to an information processing analysis. In any case, as far as we are aware, the present results are the first to link the assumption in economics that incentive is information with the Hick-Hyman law on how incentive as information is processed and also are the first to show how 
the Hick-Hyman law can buttress basic assumptions in evolutionary biology about the incentive value of information.

\section{Information Processing and the Serial Reaction Time Task}

It should not go unnoticed that our task is an analog of one that has been used to diagnose a variety of neuropsychological deficits in humans (e.g., Curran, 1997; Knopman \& Nissen, 1991; Nissen $\&$ Bullemer, 1987). In our version of this task, pigeons process information about both spatial location (Froehlich et al., 2004) and incentive (Experiment 2) according to the Hick-Hyman law. We therefore suggest that neuropsychological and neurobiological applications of the Nissen and Bullemer task would benefit from a careful distinction between the effect of a variable such as a brain lesion on reaction time versus its effect on rate of information processing. This distinction is not routinely made in the human serial reaction time literature. Indeed, we are not aware that it has ever been made. Yet, if our analogy between pigeon and human information processing in the Nissen and Bullemer task holds, neither momentary values of target spatial locations (Froehlich et al., 2004) nor momentary values of anticipated reinforcement will affect rate of human information processing, but both will affect response speed.

\section{Processing of Statistical Information and Molecular and Molar Analyses of Behavior}

Whether animals can and do track rapidly changing reinforcement likelihoods at different spatial locations has been a central question in research on instrumental behavior. If animals can track these moment-to-moment changes, one would expect to see corresponding adaptive changes in the local temporal patterning of their behavior. Furthermore, if an animal's performance were highly adaptive or even optimal at various momentary stages of a temporal pattern of changing information about reinforcement, averaging performance over the entire pattern or over different patterns would likely obscure that local optimality. Different views on this problem are partly responsible for the difference between molar and molecular analyses of choice behavior (Dinsmoor, 2001; Hinson \& Staddon, 1983; Marr, 2004; Nevin, 1969; Shimp, $1966,2004)$. The fact that information theory provides an elegant description of how pigeons track rapidly changing statistical information in the serial reaction time task may clarify a possible mechanism underlying the molecular position, according to which pigeons generally can and do track continuous changes in reinforcement probability and may even do so with nearly optimal precision. Such a position is called momentary maximizing (Hinson \& Staddon, 1983; Shimp, 1966, 2004). In particular, if local reinforcement probability is viewed as local uncertainty, then processing local reinforcement probability is processing local statistical information. A molecular position takes this local tracking into account, and a molar one intentionally does not (see Marr, 2004, and corresponding discussion). The results from Experiment 2 and from Froehlich et al. (2004) therefore constrain the generality of a molar analysis, including special cases, such as the matching law, and increase the generality of a molecular analysis.

\section{References}

Bahrick, H. P., Fitts, P. M., \& Rankin, R. E. (1952). Effect of incentives upon reactions to peripheral stimuli. Journal of Experimental Psychology, 44, 400-406.

Bennett, W. F., Fitts, P. M., \& Noble, M. (1954). The learning of sequential dependencies. Journal of Experimental Psychology, 48, 303-312.

Bolton, P., \& Dewatripont, M. (2005). Contract theory. Cambridge, MA: MIT Press.

Brainard, R. W., Irby, T. S., Fitts, P. M., \& Alluisi, E. A. (1962). Some variables influencing the rate of gain of information. Journal of Experimental Psychology, 63, 105-110.

Chase, W. G. (1978). Elementary information processes. In W. K. Estes (Ed.), Handbook of learning and cognitive processes: Vol. 5. Human information processing (pp. 19-90). Hillsdale, NJ: Erlbaum.

Curran, T. (1997). Higher-order associative learning in amnesia: Evidence from the serial reaction time task. Journal of Cognitive Neuroscience, 9, $522-533$.

Curran, T., Smith, M. D., DiFranco, J. M., \& Daggy, A. T. (2001). Structural influences on implicit and explicit sequence learning. In D. L. Medin (Ed.), The psychology of learning and motivation (Vol. 40, pp. 147-182). San Diego, CA: Academic Press.

Dickinson, A., \& Balleine, B. (2002). The role of learning in the operation of motivational systems. In H. Pashler \& R. Gallistel (Eds.), Stevens' handbook of experimental psychology: Vol. 3. Learning, motivation, and emotion (3rd ed., pp. 497-533). New York: Wiley.

Dinsmoor, J. A. (2001). Still no evidence for temporally extended shockfrequency reduction as a reinforcer. Journal of the Experimental Analysis of Behavior, 75, 367-378.

Edwards, A. E. (1979). Multiple regression and the analysis of variance and covariance. San Francisco: Freeman.

Estes, W. K. (1958). Stimulus-response theory of drive. In M. R. Jones (Ed.), Nebraska symposium on motivation (Vol. 6, pp. 35-69). Lincoln: Nebraska University Press.

Eysenck, H. (1987). Intelligence and reaction time: The contribution of Arthur Jensen. In S. Modgil \& C. Modgil (Eds.), Arthur Jensen: Consensus and controversy (pp. 285-295). New York: Falmer Press.

Frick, F. C., \& Miller, G. A. (1949). Statistical behavioristics and sequences of responses. Psychological Review, 56, 311-324.

Froehlich, A. L., Herbranson, W. T., Loper, J., Wood, D., \& Shimp, C. P. (2004). Anticipating by pigeons depends on local statistical information in a serial response time task. Journal of Experimental Psychology: General, 133, 31-45.

Garner, W. R. (1974). The processing of information and structure. Potomac, MD: Erlbaum.

Herbranson, W. T. (2000). Cognitive dissociation of memory systems: Implicit and explicit memory in pigeons (Columba livia). Unpublished doctoral dissertation, University of Utah.

Herrnstein, R. J. (1997). The matching law. Cambridge, MA: Harvard University Press.

Hick, W. E. (1952). On the rate of gain of information. Quarterly Journal of Experimental Psychology, 4, 11-26.

Hinson, J. M., \& Staddon, J. E. R. (1983). Matching, maximizing, and hill-climbing. Journal of the Experimental Analysis of Behavior, 40, 321-331.

Hull, C. L. (1943). Principles of behavior. New York: Appleton-Century.

Hunt, R. H., \& Aslin, R. N. (2001). Statistical learning in a serial reaction time task: Access to separable statistical cues by individual learners. Journal of Experimental Psychology: General, 130, 658-680.

Hyman, R. (1953). Stimulus information as a determinant of reaction time. Journal of Experimental Psychology, 65, 39-46.

Jensen, A. R. (1987). Individual differences in the Hick paradigm. In P. A. Vernon (Ed.), Speed of information-processing and intelligence (pp. 101-175). Norwood, NJ: Ablex.

Knopman, D. S., \& Nissen, M. J. (1991). Procedural learning is impaired 
in Huntington's disease: Evidence from the serial reaction time task. Neuropsychologia, 29, 245-254.

Laffont, J., \& Martimor, D. (2001). The theory of incentives: The principal agent model. Princeton, NJ: Princeton University Press.

Loewenstein, G. (2006, May 5). The pleasures and pains of information. Science, 312, 704-706.

Marr, M. J. (2004). The interpretation of themes: Opening the BaumStaddon ink battle. Journal of the Experimental Analysis of Behavior, $82,71$.

Maynard Smith, J., \& Harper, D. (2003). Animal signals. Oxford, England: Oxford University Press.

Miller, G. A., \& Frick, F. C. (1951). A statistical description of operant conditioning. American Journal of Psychology, 64, 20-36.

Nevin, J. A. (1969). Interval reinforcement of choice behavior in discrete trials. Journal of the Experimental Analysis of Behavior, 12, 875-885.

Nissen, M. J., \& Bullemer, P. (1987). Attentional requirements of learning: Evidence from performance measures. Cognitive Psychology, 19, 1-32.

Rescorla, R. A., \& Wagner, A. R. (1972). A theory of Pavlovian conditioning: Variations in the effectiveness of reinforcement and nonreinforcement. In A. H. Black \& W. F. Prokasy (Eds.), Classical conditioning II: Current research and theory (pp. 64-99). New York: Appleton-Century-Crofts.

Rosnow, R. L., \& Rosenthal, R. (2003). Effect sizes for experimenting psychologists. Canadian Journal of Psychology, 57, 221-237.

Saffran, J. R. (2003). Statistical language learning: Mechanisms and constraints. Current Directions in Psychological Science, 12, 110-114.
Shimp, C. P. (1966). Probabilistically reinforced choice behavior in pigeons. Journal of the Experimental Analysis of Behavior, 9, 443-455.

Shimp, C. P. (2004). Scientific peer review: A case study from local and global analyses. Journal of the Experimental Analysis of Behavior, 82, 103-116.

Simpson, J. A., \& Weiner, E. S. C. (Eds.). (1989). The Oxford English dictionary (Vol. 7). Oxford, England: Clarendon Press.

Sternberg, S. (1969). The discovery of processing stages: Extensions of Donders' method. Acta Psychologica, 30, 276-315.

Tolman, E. C. (1932). Purposive behavior in animals and men. New York: Appleton-Century-Crofts.

Vickrey, C., \& Neuringer, A. (2000). Pigeon reaction time, Hick's law, and intelligence. Psychonomic Bulletin \& Review, 7, 284-291.

Welford, A. T. (1968). Fundamentals of skill. London: Methuen.

Wickens, C. D., \& Hollands, J. G. (2000). Engineering psychology and human performance. Upper Saddle River, NJ: Prentice-Hall.

Yerkes, R. M., \& Dodson, J. D. (1908). The relation of strength of stimulus to rapidity of habit-formation. Journal of Comparative Neurology and Psychology, 18, 459-482.

Young, M. E., \& Wasserman, E. A. (1997). Entropy detection by pigeons: Response to mixed visual displays after same-different discrimination training. Journal of Experimental Psychology: Animal Behavior Processes, 23, 157-170.

Received June 30, 2006

Revision received October 12, 2006 Accepted October 16, 2006

\section{E-Mail Notification of Your Latest Issue Online!}

Would you like to know when the next issue of your favorite APA journal will be available online? This service is now available to you. Sign up at http://watson.apa.org/ notify/ and you will be notified by e-mail when issues of interest to you become available! 